\title{
From PraCtical to PURE GEOMETRY AND BACK
}

\author{
Mário Bacelar Valente \\ Universidade Pablo de Olavide-UPO - Espanha
}

(aceito para publicação em maio de 2020)

\begin{abstract}
The purpose of this work is to address the relation existing between ancient Greek (planar) practical geometry and ancient Greek (planar) pure geometry. In the first part of the work, we will consider practical and pure geometry and how pure geometry can be seen, in some respects, as arising from an idealization of practical geometry. From an analysis of relevant extant texts, we will make explicit the idealizations at play in pure geometry in relation to practical geometry, some of which are basically explicit in definitions, like that of segments (straight lines) in Euclid's Elements. Then, we will address how in pure geometry we, so to speak, "refer back" to practical geometry. This occurs in two ways. One, in the propositions of pure geometry (due to the accompanying figures). The other, when applying pure geometry. In this case, geometrical objects can represent practical figures like, e.g., a practical segment.
\end{abstract}

Keywords: Mathematics, History, Geometry; Euclid; Elements; Data; Optics; Meno.

\section{[Da Geometria Prática à Geometria Pura e de Volta]}

\section{Resumo}

O objetivo deste trabalho é abordar a relação existente entre a geometria prática (plana) da Grécia antiga e a geometria pura (plana) da grécia antiga. Na primera parte do trabalho, consideraremos a geometrica prática e pura e como a geometria pura pode ser vista, em alguns aspectos, como resultante de uma idealização da geometria prática. A partir de uma análise de textos existentes relevantes, explicitaremos as idealizações em jogo na geometria pura em relação à geometria prática, algumas das quais são basicamente explícitas nas definições, como a de segmentos (linhas retas) nos Elementos de Euclides. Então, abordaremos como na geometria pura "nos referimos", por assim dizer, à geometria 
prática. Isso ocorre de duas maneiras. Uma, nas proposições da geometria pura (devido às figuras acompanhantes). A outra, ao aplicar a geometria pura. Neste caso, os objetos geométricos podem representar figuras práticas como, por exemplo, um segmento prático.

Palavras-chave: Matemática, História, Geometria, Euclides, Elementos, Dados, Óptica, Mênon.

\section{Introduction}

Here, we want to address the relationship between ancient Greek practical geometry and its "counterpart", ancient Greek pure geometry. Our Greek pure geometry will consist of (parts of) two books, the Elements and the Data, both attributed to someone named Euclid. Ideally, we would like to characterize Greek practical geometry basing our account in texts, or whatever, produced previous to Euclid's works. The idea is to consider accounts that might not be "permeated" by the influence of pure geometry. There is a passage of Plato's Meno that has this characteristic (even if pure geometry was way under development by Plato's time). Section 2 of this work is an analysis of this passage of Meno, enabling to address what was Greek practical geometry previous to Euclid's time. In section 3 we consider ancient Greek pure planar geometry in relation to ancient Greek practical planar geometry. We address it in terms of the idealizations made in pure geometry. The historical analysis will enable us to see that the idealizations occur at two "levels". On one "level", we have the idealization of a practical planar surface (where practical figures are drawn) as the Euclidean plane (where geometrical objects are instantiated). On another "level", we have the idealizations of practical figures (like a practical segment) as geometrical objects (like a geometrical segment). In section 4 we will see how in pure geometry there are two senses in which we "refer back" to practical geometry. The first is in the presence of practical geometry in the propositions of pure geometry due to the accompanying figures. The second occurs when we apply pure geometry. We will see a very simple example from Euclid's Optics in which a geometrical segment is taken to represent the spatial extension of a concrete object (the "thing seen").

\section{Ancient Greek practical geometry}

By the first half of the fourth century BC, Plato envisaged that there were two kinds of geometry (and arithmetic). On one hand, we have "the art of calculating and measuring as builders and merchants use them" (PLATO, 1997, p. 446). On the other hand, we have "the geometry and calculations practiced by philosophers" (PLATO, 1997, p. 446). There is evidence that by the time of Plato there already existed what we may call pure geometry. To it, Plato refers to as the geometry practiced by philosophers. The most ancient known work of pure geometry, thought available through an indirect source, is that of Hippocrates of Chios on the quadrature of lunules, written sometime in the second half of the fifth century 
BC (NETZ, 2004). ${ }^{1}$ Besides this work, several examples of pure geometry written previous to Euclid's Elements have been handed down to us. We have, e.g., fragments from the work of Archytas on geometry (CUOMO, 2001, pp. 58-9), or geometrical proofs in Aristotle's work (see, e.g., HEATH, 1949, p. 23).

The situation with Greek practical geometry is different. The written evidence of practical geometry in ancient Greece is rather sparse. Most of what remains belonging to the Greek population in Egypt, under Rome's ruling (ASPER, 2003, p. 110). Possibly, one of the earliest (or the earliest) extant text related to Greek practical geometry is a passage in Plato's Meno. At the beginning of the passage in question, from the way the text is written we may conclude that Socrates draws a figure (or more exactly, we read a fictional account corresponding to Socrates drawing a figure):

"Socrates: Tell me now, boy, you know that a square figure is like this?-I do.

Socrates: A square then is a figure in which all these four sides are equal?-Yes indeed" (PLATO, 1997, p. 881).

The drawing that we imagine Socrates to do might well be made directly on the ground or, e.g., on a wax tablet or some other type of board (CUOMO, 2001, p. 13; NETZ, 1999, pp. 14-6). The important feature for us is that the ground or the board are taken to be practically flat. This is implicit in Socrates' drawings in the Meno. When Socrates asks: "A square then is a figure in which all these four sides are equal?" (PLATO, 1997, p. 881), it is supposed that a square was drawn in which all four sides are equal, in the sense of being drawn and measured, e.g. using a rod, such that we can consider that they are equal in practice (as measured). We will say in this case that the sides are practically equal. For this to be possible, the rod must be in contact with the surface where the drawing is being made. If the surface is too curved it would be very difficult, if not impossible, to draw four sides with the same length as determined by the rod.

Regarding what Socrates draws, we must notice that the square is identified as the figure drawn; i.e. we do not consider a square as a sort of abstract object (as in pure geometry) and the drawing as a representation of it. Being this text couched in the language of practical geometry, the square is a drawn figure. It is said that the sides are equal. Here, we must think of equality not as conceptualized in pure geometry (see below), but as the practical equality of drawn segments. We can suppose that the segments were drawn using a rod so that besides being practically straight they also have practically the same length, otherwise Socrates' interlocutor would not agree that the sides are equal ("to the eye"). Next, Socrates draws two perpendicular lines passing through the center of the square joining the middle of these sides: "Socrates: And it also has these lines through the middle

\footnotetext{
${ }^{1}$ According to Netz, "all testimonies for pre-Hippocratic mathematics must be, by my thesis, downplayed. They are either later fabrications; or, at most, they involve no more than oral teaching; or they should not count as 'mathematics"' (NETZ, 2004, p. 246).
}

RBHM, Vol. 20, n 39, pp. 13-33, 2020 
equal?-Yes" (PLATO, 1997, p. 881). Again, the determination of the middle of each side must be conceived as a practical determination made in the concrete square.

In practical geometry, we deal with (concrete) segments with lengths given in terms of some metrological system of units. For example, the rod used to draw the sides might correspond to a particular unit of length, which would imply that the length of the sides could be, e.g., one, as given in terms of this unit of length (using all of the rod's extension to draw the sides). This aspect of practical geometry is made explicit next in the text:

"Socrates: And such a figure could be larger or smaller? — Certainly.

Socrates: If then this side were two feet, and this other side two feet, how many feet would the whole be? Consider it this way: if it were two feet this way, and only one foot that way, the figure would be once two feet? - Yes.

Socrates: But if it is two feet also that way, it would surely be twice two feet? - Yes.

Socrates: How many feet is twice two feet? Work it out and tell me. Four, Socrates" (PLATO, 1997, p. 882).

Here, Socrates is not saying that the length of each side is two feet, being "foot" a unit of length (LEWIS, 2001, pp. xviii-xix). We are pretending that this is the case. We can imagine Socrates to draw in the ground a square with sizes to his convenience, not worrying to draw the square with sizes with a practical length of two feet, as drawn/measured using a rod with markings for foot (one, two, three, and so on). But the important thing for us is that the square (as the drawn figure) is being conceived in terms of measured lengths; i.e., we operate within a practical geometry. When Socrates says "[if] this side were two feet", being implicit that he is pointing to the side, and then says "and this other side two feet", again being implicit that he is pointing to another side of the figure, and then asks "how many feet would the whole be?", he is asking for the value of the area of the square. The area of the square in practical geometry is given by multiplying the measured lengths of two adjacent sides. To help his interlocutor, Socrates gives the example of the calculation of the area of a rectangle with a larger side of two feet and a smaller side with one foot. In this case, the area is two feet. To be exact we need to consider that "feet" here means a unit of area and not a unit of length; something like square feet. Returning to the calculation under consideration, Socrates asks if the value of the area would not "surely be twice two feet". Socrates asks his interlocutor to make this multiplication ("work it out and tell me"), who arrives at the result of 4 (square) feet.

Afterward, Socrates mentions that one could have a figure "twice the size of this one" (PLATO, 1997, p. 882); again, it is as if Socrates is pointing to the drawn figure. Size is the area of the figure. Being twice the size of a square with an area of 4 (square) feet, its area is 8 (square) feet. Socrates asks his interlocutor to tell him the length of the sides of the larger square. The answer given is 4 feet. By extending the original drawing of one side from 2 feet to 4 feet and drawing the other sides, Socrates draws a square with sides with 4 
feet (here, to simplify, we consider Socrates to have actually drawn initially a square with sides with 2 feet): "Now the line becomes double its length if we add another of the same length here? [...] let us draw from it four equal lines" (PLATO, 1997, p. 882). Socrates leads his interlocutor into noticing that the size of this square is not 8 but 16 (square) feet. Afterward, the interlocutor proposes the length of 3 feet for the side of a square with an area of 8 (square) feet. Socrates then draws a square with sides with 3 feet:

"Socrates: Then if it is three feet, let us add the half of this one, and it will be three feet? For these are two feet, and the other is one. And here, similarly, these are two feet and that one is one foot, and so the figure you mention comes to be? - Yes" (PLATO, 1997, p. 883).

Socrates helps his interlocutor to see that the area of the figure is of 9 (square) feet:

"Socrates: Now if it is three feet this way and three feet that way, will the whole figure be three times three feet? - So it seems.

Socrates: How much is three times three feet? - Nine feet" (PLATO, 1997, p. 883).

Until this moment everything we are doing is practical geometry. The areas of the squares are calculated by multiplying the measured length of the sides of the drawn figures, and these sides are practically equal.

Next, we deal with incommensurable sides, which can only be addressed approximately in practical geometry. ${ }^{2}$ We will be considering the diagonals of squares with 2 feet sides. Nowadays, we would say that the diagonal corresponds to the real number $2 \sqrt{2}$. However, it would not make sense to say that it is $2 \sqrt{2}$ feet, since the most we can achieve with practical measurements is to "approach" the "exact" value by taking into account subunits of the adopted length unit (e.g. if we were working with centimeters we might consider millimeters to have a more accurate measure but this is not the real number $2 \sqrt{2})$.

Since it turns out that a square with an area of 8 (square) feet does not have sides of 4 feet neither of 3 feet, Socrates somewhat rhetorically asks his interlocutor: "But on how long a line? Try to tell us exactly, and if you do not want to work it out, show me from what line" (PLATO, 1997, p. 883). As mentioned, in the context of practical geometry we cannot tell "exactly" or "work it out" how long the line is (i.e. the length of the side of a square with a size of 8 (square) feet). The most we could do is to give an approximate measure of this length. That is why Socrates also tells his interlocutor to show him from what line a square with an area of 8 (square) feet can be determined. Socrates then draws a square made of four squares with sides with 2 feet. In this way, each of the squares was an

2 The existence of approximate calculations in practical geometry is attested, e.g., in some Old Babylonian mathematical procedural texts (see, e.g., $\square$ ØYRUP, 2002, pp. 268-72 \& 297-8). 
area of 4 (square) feet. Socrates then draws 4 diagonals, one for each square. The diagonals can be seen as the boundary of another square inscribed in the larger one (see figure 1).

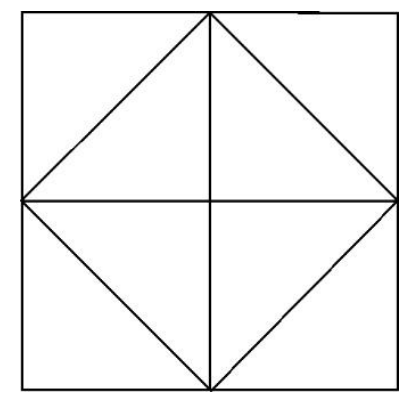

Figure 1. Possible drawing being made by Socrates corresponding to his comments in this part of the Meno. The larger square has sides with 4 feet and an area of 16 (square) feet. The inscribed square has an area of 8 (square) feet.

Socrates calls the attention of his interlocutor that each diagonal divides each square into two parts (with the same area): "Within these four figures, each line cuts off half of each, does it not?" (PLATO, 1997, p. 885). Then Socrates leads his interlocutor into noticing that the inscribed square is "made of" four triangles, while one small square is made of two triangles. In this way, the area of the inscribed square is double that of one square (which is 4 square feet). This means that its area is of 8 (square) feet:

"Socrates: How many of this size are there in this figure? - Four.

Socrates: How many in this? - Two.

Socrates: What is the relation of four to two? - Double.

Socrates: How many feet in this? - Eight" (PLATO, 1997, p. 885).

Since we are working within practical geometry, Socrates cannot refer to the diagonals that are the sides of the inscribed square in terms of a unit of measure. He avoids that as follows:

"Socrates: Based on what line? - This one.

Socrates: That is, on the line that stretches from corner to corner of the four-foot figure? - Yes" (PLATO, 1997, p. 885).

Socrates identifies the line in the figure but does not mentions its length, since he cannot. However, he still dwells in practical geometry: it is the line that stretches from corner to corner of a drawn figure with sides taken to have 2 feet corresponding to an area of 4 (square) feet.

In ancient Greek practical geometry (as portraited in the passage of the Meno considered), a geometrical figure (e.g., a square) is identified with the drawing made on a flat surface. In the case under consideration, of a square, to be called so, a drawing must 
consist on four (practically) equal segments, e.g., as measured with a rod or a rope and have a (practically) right angle between adjacent segments (a feature left implicit in the Meno). These are general features of ancient practical geometry (Greek or others), not an idiosyncrasy of the passage of the Meno under discussion. For example, in a third century BC papyrus, we see other cases of ancient Greek practical geometry (CUOMO, 2001, pp. 70-2). There, one considers several "plots of land" (taken implicitly to be flattened surfaces), their shape (e.g., in one case a rectangular shape and in another case a circular shape), and several measures and calculations related to them; e.g., the area of a surface given in the unit of square cubits, or the length of distinct segments (in the unit of cubits). In one of the problems, the lengths of the segments forming a square inscribed in a circle, which are to be determined, are even referred to in terms of "measurements": "four segments, [what are] their measurements?" (CUOMO, 2001, p. 71).

\section{Ancient Greek pure (planar) geometry}

As we have seen in the previous section, initially, Socrates draws a square. This drawing is made on a (practically) flat surface, e.g., the ground (if it is sufficiently flat), a wax tablet, or some other type of board (CUOMO, 2001, p. 13; NETZ, 1999, pp. 14-6). Here, we face the first of the idealizations of ancient Greek planar pure geometry, at least as rendered in Euclid's work: the Euclidean plane. The Euclidean plane is never mentioned directly in Euclid's work; ${ }^{3}$ it is left implicit as a background. As Mueller notices:

"Normally, when plane geometry is developed as an independent subject, it is taken for granted that all objects considered lie in a single plane, which never has to be mentioned" (MUELLER, 1981, p. 208).

We can consider the Euclidean plane as an idealization of the concrete planar board where drawings are made (TAISBAK, 2003, p. 19). ${ }^{4}$ We can think of the Euclidean plane as a bi-dimensional space with absolute positions. ${ }^{5}$ It is a space where we can put

\footnotetext{
${ }^{3}$ In the Elements, Euclid mentions the notion of surface which is not to be confused with the Euclidean plane. A surface is a sort of a planar figure since it has no depth (definition 5) and its extremities are lines (definition 6), and the extremities are a boundary (definition 13), and a figure is contained by boundaries (definition 14). Also, the surface is, sort of, made up by straight lines (segments), since, according to definition 7, "a plane surface is a surface which lies evenly with the straight lines on itself" (EUCLID, 1956, p. 153). As we will see, the Euclidean plane is a sort of space where points, segments, circles, or other planar figures are positioned.

4 Taisbak actually writes that the Euclidean plane "is an abstraction from physical boards" (TAISBAK, 2003, p. 19). We prefer the term idealization for a couple of reasons: (a) the term abstraction is well-known as adopted in Aristotle's philosophy of mathematics (see, e.g., BÄCK, 2014). Here, we adopt a historical approach. It should be neutral regarding, e.g., the adoption of an Aristotelian view or a Platonic view; (b) The term idealization seems to be adequate regarding the Euclidean plane or, e.g., geometrical segments. For this last case, the term idealization seems more appropriate as we will see in the text.

${ }^{5}$ The Euclidean plane would share with the Newtonian absolute space the notion of absolute position. It is beyond the scope of this work to address similarities and differences between these two constructs. On Newton's notion of absolute space see, e.g., JAMMER (1993, pp. 95-116).
} 
geometrical objects in specific positions. This can be contrasted with a relative space where only the relative position of objects is relevant. ${ }^{6}$ In particular, as we will see in more detail below, geometrical points are determined by their (absolute) positions in the Euclidean plane. When a geometrical construction starts, be it in Euclid's Elements or Data, some initial objects are given according to explicit or implicit postulates of the Elements. ${ }^{7}$ Points, segments, and circles are given, i.e. put at our disposal in the Euclidean plane. A point is given in position, i.e. it is "put" at an absolute position of the Euclidean plane; and segments and circles are "drawn" or "described" in the plane according to the postulates of the Elements, which in the language of the Data translates in saying that they are given in position or magnitude. How are points, segments, or circles instantiated in the Euclidean plane? i.e., how are they given or put at our disposal in the plane? This is made by The Helping Hand. According to Taisbak:

"The Helping Hand [is] a well-known factotum in Greek geometry, who takes care that lines are drawn, points are taken, circles described, perpendiculars dropped, etc. The perfect imperative passive is its verbal mask: 'Let a circle have been described with center A and radius AB', 'let it lie given' [...] Always The Helping Hand is there first to see that things are done [...] There is no magic involved, though; The Helping Hand can do only such work as is warranted by postulates or propositions" (TAISBAK, 2003, pp. 28-9).

There are then rules for how geometrical objects can be instantiated in the Euclidean plane, and this is taken to be done ex nihilo by The Helping Hand.

To better understand the Euclidean plane, we need to consider the basic geometrical objects (points, segments, and circles) that are positioned in it. Let us start by considering the geometrical segment (straight line) as defined in the Elements. According to definitions 2 to 4: "A line is breadthless length. The extremities of a line are points. A straight line is a line which lies evenly with the points on itself" (EUCLID, 1956, p. 153).

A geometrical segment can be seen as an idealization of a concrete segment: a rod, a stretched rope, or, e.g., a drawing in a wax tablet made using a rod. The idealizations that are manifested in the geometrical segment are: its continuity; the "perfect" straightness; the lack of depth; the breadthlessness; and what we will call the exactification of length. We

\footnotetext{
${ }^{6}$ The distinction between spaces with absolute or relative positions is made in the context of physical theories of space, i.e. in a quite different context. However, it might be helpful to better understand the implication of having absolute positions in the Euclidean plane. On physical absolute versus relative spaces see, e.g., EARMAN (1989).

${ }^{7}$ According to Taisbak, besides the postulates 1 to 3 of the Elements, we have to consider to be implicit a postulate 0 for points: "any point may be (taken and) appointed given" (TAISBAK, 2003, p. 94). In fact, this "postulate" is somewhat implicit in postulate 1: "to draw a straight line from any point to any point" (EUCLID, 1956, p. 154). For this to be possible first points must be given. Regarding the term "given", the language of the "givens" is more fully developed in the Data than in the Elements. According to Knorr, "the Data is a complement to the Elements $[\ldots]$ each of its theorems demonstrates that a stated term will be given on the assumption that certain other terms are given [...] only in rare instances does the Data present a result without a parallel in the Elements" (KNORR, 1986, p. 109).
} 
can see that the definition of segment is made by explicit (or implicit) reference to the idealizations being made. As such it is defined in relation to what is being implicitly idealized: a practical segment. It only makes sense a definition in terms, e.g., of a lack of depth (implicit in planar geometry) and breadthless, in relation to something that has depth and breadth (we can only have a notion of breadthless when having first a notion of breadth and this, like depth and length, arises in relation to concrete things).

The counterpart of the concrete continuity that we see, e.g., in a rod or a rope is the geometrical continuity of lines. This continuity is manifested, e.g., in proposition 1 of book 1 (I.1) of the Elements, when it is taken for granted that two lines (in this case two circumferences) cross at a point. The continuity of segments (or other geometrical objects) is implicit in their treatment as magnitudes. According to Mueller:

"The most appropriate interpretation of magnitudes in the Elements involves construing them as abstractions from geometrical objects which leave out of account all properties of those objects except quantity: i.e. length for lines, area for plane figures, volume for solids, size, however characterized, for angles" (MUELLER, 1981, p. 121).

If we adopt an Aristotelian view (or take it to be implicit in Euclid's notion of magnitude), the magnitude is the "aspect" of geometrical objects related to spatial extension (or saying a bit differently, related to the spatial extension of their concrete counterparts), and as such its "most significant property [...] is that it is continuous" (TAISBAK, 2003, p. 30; see also HEATH, 1949, p. 45).

This continuity has an implication regarding the Euclidean plane. If it is not in some sense a continuous space, how could continuous geometrical objects be instantiated in it? A (continuous) segment is given in position. All of the points evenly on it are given in position. There is so to speak a continuum of positions in the Euclidean plane. This again can also be seen as compatible with the Euclidean plane being an idealization of practically planar boards.

In this respect, while these boards are small (see, e.g., CUOMO, 2001, p. 13, figure 1.4), the Euclidean plane has no defined boundaries. It seems that we are to consider it as having a size larger than whatever geometrical objects are instantiated in it. However, by postulate 2 of the Elements, a segment can be extended indefinitely (EUCLID, 1956, p. 154). Might we assume that the same happens with the Euclidean plane, or simply that this worry was not taken into account? Whatever the case, it seems that the undefined boundary (or lack of meaning of this idea) of the Euclidean plane is compatible with practical geometry, in which a drawing is always made in a circumscribed surface. That is, the drawings are made to fit the practical planar board. If e.g., we draw a segment up to the boundary of the board, and "imagine" that it goes on and on (somewhat like in postulate 2), we are in fact already going beyond the realm of practical geometry.

Regarding the "perfect" straightness of segments, possibly the most elucidative "definition" is that of Heron: "a line stretched to the utmost" (cited in HEATH, 1949, p. 93). Here, we see a parallel between the geometrical line and a stretched rope. This makes 
the idealization understandable even if unrealizable with concrete objects. The straightness of the line is a sort of non-realizable "limit" of concrete straightness.

Let us now consider the lack of depth. To be positioned on a Euclidean plane, any geometrical object must not have a depth. Since we can see the Euclidean plane as an idealization of a practically planar board, we can also consider as our concrete line (e.g., a segment or a circumference) one drawn in a board. As it is, the drawn line is as if having no depth. At least "to the eye". We would need, e.g., a microscope to see the "rugosity" of the board and that the line is made of material deposited on the board having different depths along the drawing. The idealization of a line as having no depth is compatible with the concrete drawings on practical planar boards.

More cumbersome might seem to be the idea of a line as a "breadthless length" (EUCLID, 1956, p. 153). Again, this definition is compatible with practical geometry. When Socrates draws a square, its sides are equal with respect to their length (as measured with, e.g., a rod). There is no issue as to measuring the breadths of these lines. They are very small in relation to their lengths. Only the straightness and length of the lines are relevant for the drawing of a practical square. The breadth could be a bit larger or smaller; that is irrelevant. This is taken into account in the definition of the geometrical line; as mentioned by Harari, "a line is measurable with respect to its length, while it is nonmeasurable with respect to its breadth" (HARARI, 2003, p. 18). More in terms of the language of pure geometry, we might say that the line is a magnitude only with respect to its length. As such, what to do with the non-measurable breadth? Here, enters the idealization. We use a term which simply does not make sense in relation to concrete objects (even a line drawn in a board). We say that the geometrical line is breadthless. Like in the case of the "perfect" straightness we can envisage this concept indirectly through a sort of limiting procedure. We might imagine lines with smaller and smaller breadths until we barely see the last one. We could imagine our breadthless line to come just next - the "first" "unseen" line.

The elements of idealization inbuilt in geometrical objects when considered in relation to concrete objects are quite consistent with practical geometry. In it, we have practically continuous objects (e.g. ropes or drawings of lines in boards), a practical straightness, the dispense of the depth in planar boards and drawings made in them, and the practical irrelevance of the breadth of lines, which we ignore in practical geometry.

The issue of the exactification of lengths is different. In practical geometry, length must be measured using a system of units and material embodiments of the units (e.g. a unit-measuring rod). The length of drawn lines refers back to these measurements. We have seen that even in the case of the diagonal of a square that was incommensurable with the sides, Socrates describes it indirectly through an area unit (which is calculated from the measure of the sides). In pure geometry, we can dispense with measures. This is done, e.g., in the early books on planar geometry in the Elements. How do we do this? We take the concrete extension and have as its counterpart in a geometrical object its feature as a magnitude. We do not have to consider the counterpart of the concrete act of measuring one concrete length using another concrete length (e.g., when we measure the length of a drawn segment using a rod). As a magnitude, a segment has a length by itself. This length is exact; 
it does not depend on measuring procedures. It belongs to the geometrical object, not depending on its measurement by an external element.

One of the implicit postulates of Euclid's geometry is that we can be given, by The Helping Hand, different segments with unequal length. For example, two segments that are clearly unequal can be put at our disposal in the Euclidean plane. Why "clearly"? As we can see, e.g., in proposition 3 of book 1 (I.3) of the Elements, it is taken for granted that it is evident which one is larger (EUCLID, 1956, pp. 246-7). The situation regarding equal segments requires more care. It seems that we cannot be given directly two equal segments. Maybe because two practical segments might seem "to the eye" equal but not being really so? The matter of fact is that, in the Elements, to have two equal segments we need to construct one segment after we are given another (EUCLID, 1956, p. 244). ${ }^{8}$ How in the Elements and the Data, can we have established the equality of segments? This comes with the geometrical object called circle. Again, like in the case of a geometrical segment that is conceived in the Elements in terms of an idealization of a concrete segment, the geometrical circle is also in a "relation of idealization" with respect to the circle of practical geometry. The practical circle is, e.g., drawn with a compass, in which one of its legs is fixed in the board on what we call the center of the circle and the other draws the circumference. We can consider the circle as the figure circumscribed by the circumference. A feature of a practically drawn circle is that its radii (the segments connecting the center to the circumference) are practically equal; i.e. they are equal as measured, e.g., with a rod. The geometrical circumference, like other geometrical objects, is continuous, lacks a depth (a circle is a planar object), and is breadthless. Also, it is postulated that its radii are exactly equal. Here, we assist at the exactification of length at its most. We are not simply saying that the length of a segment is exact, we are saying that we can be given countless segments that are exactly equal; i.e., that have the same exact length. So, if we are given a segment and want to construct another one that has the same length, this can be done by using this segment as the radius of a circle (as it is done in proposition I.1 of the Elements; see EUCLID, 1956, pp. 241-2). ${ }^{9}$

Let us now address the geometrical object called point. To Plato, "point" is a geometrical fiction (HEATH, 1949, p. 80). In fact, Euclid's definition is that "a point is that which has no part" (EUCLID, 1956, p. 153). How can we understand this definition? Again, it relates to the feature of measurability. As mentioned by Harari, "a point is characterized as a non-measurable entity, as it has no parts that can measure it" (HARARI, 2003, p. 18). This implies that a point cannot be considered in terms of magnitude. But, since it is by definition a non-measurable object, how can we consider it in terms of a relation of idealization between geometrical objects and concrete objects of practical geometry? If we recall the definition of geometrical segment, it is breadthless. Let us

\footnotetext{
${ }^{8}$ As we will see below, in the Data there is an alternative procedure to be given a segment which we take to be equal to others, even if these are not actually put at our disposal in the Euclidean plane.

9 We will also adopt the term "relation of idealization", in particular when wanting to stress that by conceiving a geometrical figure in terms of the idealization of a concrete figure this implies a relation between them. In this way, we might also say that the concrete circle is in a relation of idealization with the geometrical circle, which is to be understood as just indicated.
} 
imagine that two geometrical segments cross each other perpendicularly. They would not be crossing their "lengths", they would be crossing their "breadthless" breadths. We take the segments to be continuous. This implies (by definition or postulation if you will) that when crossing, there must be something there in the crossing. Whatever this something is (as a geometrical object), as the example of the crossing of two perpendicular segments shows, it cannot have a magnitude "attached" to it. We call it a point and say that it is that which has no part. We think that points are no more geometrical fictions than any other geometrical object. Points as non-measurable objects are compatible with the relation of idealization existing between practical segments and geometrical segments. In fact, if we wish we can see the geometrical point as conceived as an idealization of the surface of the crossing of practical segments (or lines). This surface of the crossing is not considered as a practically measurable surface. It consists of the crossing of the breadths of two practical segments (or lines), which are not considered in terms of length measurements.

There are several properties attributed to points as geometrical objects, some made explicit in the definitions, others implicit and revealed in the propositions. In particular, according to definition 3, "the extremities of a line are points" (EUCLID, 1956, p. 153), and, according to definition 4, "a straight line is a line which lies evenly with the points on itself" (EUCLID, 1956, p. 153). Implicit in postulate 1 is that points can be given in the Euclidean plane; when this is done, if necessary, we can rely on The Helping Hand to "draw a straight line from any point to any point" (EUCLID, 1956, p. 154). Another feature, that we already mentioned, which is somewhat implicit in the Elements but explicit in the Data, is that points are given in position. For example, in proposition I.2 of the Elements we have to place a segment equal to a given one at a given point. The initial segment and the point are given in position in the Euclidean plane. We have to construct a segment equal to the one given at a particular (absolute) position of the Euclidean plane: the (absolute) position where the point is located. As mentioned in the Data, "given in position is said of points and lines and angles which always hold the same place" (TAISBAK, 2003, p. 17). Also, as mentioned, a line (straight or not) has points on itself. This means, e.g., not only that when lines cross, they do so at a point, this also implies that we can "pick" a particular point of a line; i.e., we identify in position a particular point "belonging" to a line. For example, in proposition I.5 of the Elements, "a point F [is] taken at random on [the segment] BD” (EUCLID, 1956, p. 251).

While points are given in the Euclidean plane only in position, segments, geometrical figures (like the circle), and angles can also be given in magnitude. For our purpose, it will suffice to address segments. Segments are always given in position in the Euclidean plane, like any other geometrical object. However, when the relevant feature is not the particular position of the segment but that it has the same "magnitude" as another segment, the position is not the relevant property of the segment. According to Taisbak:

"The [Euclidean] plane is a scene into which the Geometer may put geometrical objects, particularly points and lines, which in turn will produce figures. Some of these points and lines are labelled given in position and may not hop around: they are supposed to be identified from 
now on. Other lines and figures do have position, but are 'only' given in magnitude so that any object equal to a given may serve as well" (TAISBAK, 2003, p. 96).

But what exactly does it mean, e.g., for a segment to be given in magnitude? According to definition 1 of the Data, "given in magnitude is said of figures and lines and angles for which we can provide equals" (TAISBAK, 2003, p. 17). What this means is, as Taisbak puts it, that, e.g., "a [segment as a] magnitude is given if there is a (latent) [segment as a] magnitude equal to it" (TAISBAK, 2003, p. 95). To see how a segment can be given in magnitude or "just" in position let us consider a couple of theorems of the Data. Theorem 30 states that "if from a given point to a straight line given in position a straight line be drawn making a given angle, the line drawn is given in position" (TAISBAK, 2003, p. 103). We are given by The Helping Hand a straight line (a segment) BC and a point A; these are positioned in the Euclidean plane and as such have a position: they are given in position. Let a point $\mathrm{D}$ be taken on line $\mathrm{BC}$ (this is implicit in theorem 30). After picking a point labeled as D, The Helping Hand provides us with a straight line (a segment) having as extremities the points A and $\mathrm{D}$ : "let the straight line $\mathrm{AD}$ have been drawn" (TAISBAK, 2003, p. 103; see figure 2). This segment $A D$ is given in position. Its position in the Euclidean plane is fixed by the absolute positions of the points A and D. There is no other segment (present or latent) in the Euclidean plane equal to $\mathrm{AD}$ such that we might consider $\mathrm{AD}$ to be given in magnitude.

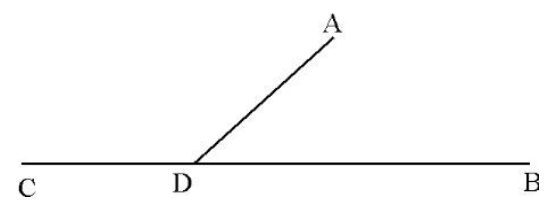

Figure 2. Version of the drawing in theorem 30 of the Data.

Let us now see how a segment can be given primordially in magnitude (as mentioned any geometrical object given in magnitude is also given in position). Theorem 31 states that "if from a given point a straight line given in magnitude be drawn to meet a straight line given in position, the line drawn is also given in position" (TAISBAK, 2003, p. 104). For us, the relevant feature of this theorem is that it shows how a segment is given in magnitude. How is it that a segment given in magnitude is "drawn" (i.e. instantiated in the Euclidean plane as if by some procedure; in this case, a counterpart of an actual drawing in a board)? It is as if it is given in magnitude previous to be given (put at our disposal) in the Euclidean plane? That is not so. Looking at figure 3 it is very similar to figure 2 . If we were considering it in terms of an actual drawing in practical geometry, in the first case we only use, e.g., a straightedge, which does not provide any indication of the length of the segment. However, in the second case, it is made explicit in the figure, but not in the text, that the segment is determined using, e.g., a compass. This corresponds in pure geometry to consider that an arc of a circle is "described" in the Euclidean plane. Now, as we have seen, the circle is the "bearer" of the exact equality of segments. All radii of the circle are equal. 
This means that the latent radii, not "drawn" in the Euclidean plane, serve as the equals being provided such that we may consider the segment "drawn" as given in magnitude. Evidently, as is showed in theorem 31, being given in magnitude means also that the segment is given in position.

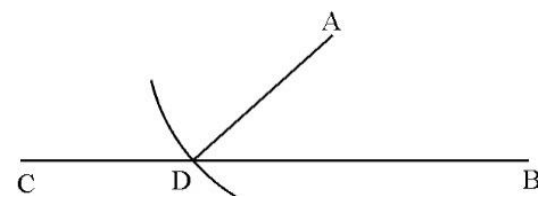

Figure 3. Version of the drawing in theorem 31 of the Data.

There is one final aspect related to the Euclidean plane and the role of The Helping Hand which we need to address. According to Taisbak, it is not just that points, segments, and even circles are put at our disposal in the Euclidean plane by The Helping Hand, which also takes care of whatever construction (drawings) need to be made. Taisbak considers that geometrical figures can be seen in some way as "constituted" outside the Euclidean plane and only afterward positioned in it. In proposition VI.14 of the Elements, we are told about two equal equilateral parallelograms. Afterward, we are told that these parallelograms are laid down (in the Euclidean plane, which of course is left implicit) along two of their sides. According to Taisbak:

"At the opening, the parallelograms are not yet placed, only thought of and named; then (without any suggestion about how to do it, but we trust The Helping Hand) they are put into the Plane to make a useful gnomon" (TAISBAK, 2003, p. 94).

An alternative to conceiving geometrical objects "only in thought", is to consider that each can be conceived in terms of a "private" Euclidean plane for each one. We take them to be constituted in a previous Euclidean plane previous to being "laid down" in the Euclidean plane in relation to which the demonstration is being made. This is a cumbersome option. There is no evidence on the Elements or the Data for this possibility. Also, it is contrary to what is done in practical geometry. In our view, the simplest option, that is faithful to the Elements, is to consider that, how it is written, the text is a figure of speech. We can only have the given, and "draw" or "describe" from which is given, as it is stipulated in the Elements. To say that the parallelograms are such and such and afterward to say that they are laid down in this or that way might be seen as a "shortcut" to actually constructing the figure following what is postulated in the Elements.

Altogether, we think that we may consider that in (planar) pure geometry we have the Euclidean plane with the properties mentioned (which is conceived as an idealization of practically planar boards) and objects that can be given in it, in particular points and segments, having an absolute position in the plane. From these given objects, others can be instantiated in the plane according to the rules of the Elements. We do not consider that we 
actively do this. There is The Helping Hand which puts the necessary elements at our disposition as necessary in the proposition under consideration. These objects, like the Euclidean plane itself, are conceived in the Elements in terms of idealizations from practical geometry.

\section{From pure geometry back to practical geometry}

Let us consider the proposition I.1 of Euclid's Elements. The enunciation is as follows: "On a given straight line to construct an equilateral triangle" (EUCLID, 1956, p. 241). At the beginning of the construction part, we are told: "Let AB be the given finite straight line". This must be read taking into account the accompanying figure (see figure 4 , left or right).
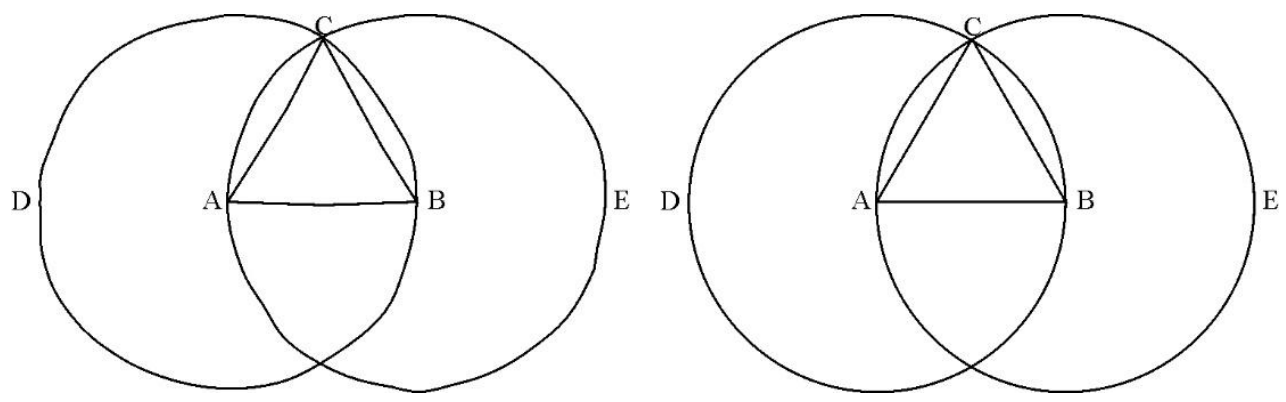

Figure 4. Two versions of the figure in I.1 of the Elements: (left) a representation of the composed geometrical object instantiated in the Euclidean plane (we follow Barceló in this drawing; see BARCELÓ, 2018, p. 13); (right) a representation or an actual practical figure that is in a relation of idealization with the geometrical object.

What is the relation between the stroke (or segment) drawn in the figure, the text, and the geometrical segment? From what we have seen, the given finite straight line (the given segment) must be understood as a segment instantiated by The Helping Hand in the Euclidean plane. While in the enunciation it is not named, in the following text (and accompanying figure) it is named $\mathrm{AB}$, which identifies, for us, in the text and figure, the given segment. But why should we take the drawn stroke (named $\mathrm{AB}$ ) to be related somehow to the geometrical segment? Evidently, the text says that this is so. But why should we accept the actual physical drawing? We take this drawing to be a representation of the segment given in the Euclidean plane, which is identified (for us) by naming it $\mathrm{AB}$ in the text and the drawing. There is an intentional feature here; one decides that this stroke represents the given segment. But is this a good representation (and what makes a good representation in ancient Greek planar pure geometry)? Without going too much into the issue of representations, in the case under consideration, we are trading with the notion of resemblance between what represents and what is being represented. ${ }^{10}$ The stroke does not

\footnotetext{
${ }^{10}$ In relation to intentional features of representations and the notion of resemblance in relation to representations, see, e.g., ABELL (2009), KULVICKI (2014), BLUMSON (2014). Here, we are not imposing a philosophy of
} 
have directly a resemblance with the geometrical segment. The latter is not a physical figure or object. ${ }^{11}$ What the stroke can be taken to represent is a practical segment. But are these not the same thing? We must consider a practical segment as a segment drawn according to accuracy and metrological standards, which are not ad hoc but depend on the historical moment and the purpose for which is drawn the practical segment. A practical segment in the times of the Meno might be a segment drawn with a straightedge and whose length is measured with a rod or a rope in a system of units, e.g., equivalent to the order of the millimeter. Nowadays we might need, for a particular purpose, a practical segment to be drawn more accurately. This depends on the available technology (for drawing) and metrology (to determine, e.g., its straightness, its length, and its breadth). A very simple criteria, e.g. in relation to didactical presentations (like in a certain sense the one in the Meno is), for a segment to be taken to be a practical segment could be that "to the eye" it looks straight and we have (or could have) a measure associated to its extension. In the same way, a circle figure might be taken to be a practical circle if it looks like it; i.e. it looks "to the eye" that the radii are of the same length. Again, we would need to have (or at least have the possibility of having), e.g., a measure of the length of the radius. In this way, figure 4 (right) could be taken to be a practically drawn figure. In this case, a composite figure made of three practical segments and two practical circles.

On the other hand, figure 4 (left) does not qualify as a practical figure; it is a drawing representing a practical figure like figure 4 (right). For example, having a practical segment (or an "idea" of what it is), we can intentionally take some stroke to represent it. It might be a good or bad representation in the sense or resembling more or less a practical segment. Again, there is nothing ad hoc here. We can measure the stroke and provide some numerical value to characterize, e.g., its departure from straightness or its breadth.

What we do afterward is, intentionally, to take the stroke that represents the practical segment as if representing directly a geometrical segment. We, so to speak, forget that the practical segment is a sort of intermediary in the representation relation between the stroke and the geometrical segment. This can be done first of all because the geometrical segment is conceived in terms of an idealization of the practical segment. If there was not this "connection" it would be meaningless to say that a stroke represents a geometrical segment; we might as well choose a red dot as representing the segment or whatever drawing or symbol we wish. Second, the intentional feature of the representation relation gives us the liberty to decide, taking into account the relation between practical and geometrical segments, that since the stroke can be taken to represent a practical segment it

representation to our historical treatment. We just adopt key notions of "representation theory" like "representation" itself, "intentionality", and "resemblance", to help to make explicit what Euclid actually does. That is, we use these terms as a mean to make more understandable and explicit the interplay of practical geometry and pure geometry in the propositions of pure geometry (e.g., in I.1 of the Elements), or in propositions of applied pure geometry (like proposition 1 of the Optics).

${ }^{11}$ The geometrical segment is an "abstract entity" that is instantiated in an abstract "Euclidean plane". If we try to think of it in terms of a physical segment it would be impossible to see it since it is breadthless (recall the metaphor of the geometrical segment being a sort of the counterpart of a limiting procedure of concrete segments with lesser and lesser breadth). In this way, we consider that a geometrical segment cannot resemble or be resembled by a practical segment. 
can also be thought of as a representation of the geometrical segment without making explicit the indirect character of this representation. That is, we intentionally consider the stroke as representing the geometrical segment.

It could also be the case that instead of a representation of a practical segment or figure, like in figure 4 (left), we have directly a practical segment or figure, like in figure 4 (right), if taken to be a practical figure. In this case, we, intentionally, take the practical segment as representing the geometrical segment. We can also not make a clear distinction between a stroke and a practical segment if they are not different "to the eye", and simply say that the drawn segment represents the geometrical segment. The same applies to the complete composite figure. This means that figure 4 (right) qualifies both as a representation of a practical figure or directly as a practical figure. Not making a clear distinction between them, we simply say that the drawn figure represents the geometrical figure instantiated in the Euclidean plane.

In proposition I.1 of the Elements, after having been given the segment identified as $\mathrm{AB}$ (and rephrasing what needs to be done in terms of this segment), it follows in the text of the proposition: "With center A and distance $\mathrm{AB}$ let the circle $\mathrm{BCD}$ be described" (EUCLID, 1956, p. 241). We take the circle as being "described" in the Euclidean plane (by The Helping Hand); that this can be done follows from postulate 3 (EUCLID, 1956, p. 154). The point in the Euclidean plane identified in the text and figure as A is taken to be the center of a circle in which its radius is the geometrical segment previously given in the Euclidean plane (identified as $\mathrm{AB}$ in the text and figure). We see that the geometrical objects are put at our disposal in the Euclidean plane sequentially by The Helping Hand (following the text indications). We have access to them, indirectly, in the text and drawing, which refers back to what is happening in the Euclidean plane (as we follow the text). Again, the text and figure enable to identify (for us) the circle instantiated (by "describing" it) in the plane. It is named $\mathrm{BCD}$ (here, point $\mathrm{C}$ is only clearly identified after drawing a second circle, as the point where the two circles intersect). From the perspective of the Data, the given segment (named $\mathrm{AB}$ ) is given in position, not in magnitude. When drawing the circle from the segment given in position, all its radii are equal to the given segment. In this way, the circle provides equals to the given segment and we might consider, afterward, that it is given in magnitude. However, initially, the segment is only given in position. So, strictly speaking, we must consider the circle as "just" given in position (as defined, a circle is given in magnitude when first the segment is given in magnitude, which is not the case here; see TAISBAK, 2003, pp. 34 \& 229). After a first circle has been described in the Euclidean plane, a second one is described also: "again, with center B and distance BA let the circle ACE be described" (EUCLID, 1956, p. 241). The second circle instantiated in the Euclidean plane is identified in the text and figure as ACE. Its center is the point of the plane identified in the text and figure as B. Its radius, like in the previous case, is the given segment (named in the text and figure as $\mathrm{AB}$ ).

The crucially important point of the Euclidean plane named C (for us) is again identified in the text and the accompanying figure. According to the text, $\mathrm{C}$ is the point "in which the circles cut one another" (EUCLID, 1956, p. 241). As we have seen, the Euclidean plane is continuous (there is a "continuous of positions" in it) and the geometrical objects 
that can be considered as magnitudes are continuous (a point is not continuous, it is as defined "that which has no parts"; it only has a position in the Euclidean plane). The circles are instantiated in the Euclidean plane in such a way that they cross each other. Like in the case of the intersection of segments, since circumferences are breadthless, it is considered that circles cross at a point. This is represented in the figure by identifying the intersection of the drawn circles as "C" (implying that it is taken to represent a geometrical point). Finally, the equilateral triangle is instantiated in the Euclidean plane by instantiating the geometrical segments identified in the text and figure as CA and CB.

As mentioned, what we have written regarding the geometrical segment named $\mathrm{AB}$ (represented by a stroke in figure 4 left, or what can be regarded as the practical segment $\mathrm{AB}$ in figure 4 right), also applies to the case of the circles $\mathrm{BCD}$ and $\mathrm{ACE}$, to their intersection (point C), and to the geometrical segments CA and CB.

Regarding figure 4 right, we can consider it as a practical figure, and we can, intentionally, take this practical figure as a representation of the (composite) geometrical figure. Again, like in the simpler case of a practical segment, we cannot say that the figure 4 right resembles the geometrical figure instantiated in the Euclidean plane, but we, intentionally, take the figure 4 right as a representation of the geometrical figure due to the relation of idealization established between them.

We can also go the other way around. When in the context of applied geometry, i.e. applying pure geometry in the description of physical phenomena, we can consider a geometrical object as representing a concrete magnitude; e.g., a geometrical segment as representing a rope or a practical segment drawn in a board or the ground. Again, the intentional feature of representations enables us to have this elasticity in what we take to represent and what we take to be represented. Since we have a relation of idealization between the geometrical segment and a practical segment, we can decide that the geometrical segment represents in the Euclidean plane the practical segment. Existing a resemblance of concrete objects to the practical segment, we can maintain the view that a geometrical segment represents the concrete object (in what regards its spatial extension). If this is a good or bad representation depends on the context. ${ }^{12}$ For example, in Euclid's Optics, an eye is taken to be represented by a point (BURTON, 1945, p. 357). In the case of the planar practical geometry, we might consider the eye to be represented as an arc of a circle or even a segment. In the case of the Optics, the eye is very small when compared to the distance to the seen object (represented as a large segment). This makes it possible to make the "approximation" of treating the eye, in the Euclidean plane, as a point. ${ }^{13}$ We could say that the point approximates in the plane a segment (of the plane) that represents the concrete eye. Evidently, we can also simply say that the geometrical point represents the eye since we do not have to consider the above-mentioned reasoning as implicit in the Optics. The point might have been taken "directly" as representing the eye.

12 For a simple example of how the mathematical context is relevant in relation to the representation of a geometrical segment see BARCELÓ (2018, pp. 32-3).

${ }^{13}$ In the case of the Optics, we consider that it is fair to say that this is a good representation, since, simply, the propositions of the Optics depend on the "approximation" of treating the eye as a geometrical point (see BURTON, 1945). 
There are then two ways in which pure geometry "refers back" to practical geometry. In propositions, usually, the accompanying figures represent composed geometrical objects. As we have seen, this is a simplified way of addressing how the representation relation is established. Properly speaking a figure represents a composed practical figure which is in a relation of idealization with the composed geometrical object. Also, it can be the case that the figure "conflates" with the practical figure; i.e., the drawn figure can be itself a practical figure. In this case, the practical figure is representing the geometrical object. In the proposition, with the accompanying figure, we "refer back" to practical geometry (in some form or another of the above-mentioned), while we are addressing geometrical objects. There is a second way in which we can "refer back" to practical geometry. It is when applying pure geometry. In this case, a geometrical object (e.g., a segment) is taken to represent a concrete object (e.g. a practical segment).

In this situation, e.g., part of the representational role of a figure of a proposition of Euclid's Optics has a sort of self-referential character. We have the case in which a practical segment (in the drawn figure) is taken to represent a geometrical segment that represents a practical segment; or more precisely, the length of a concrete object.

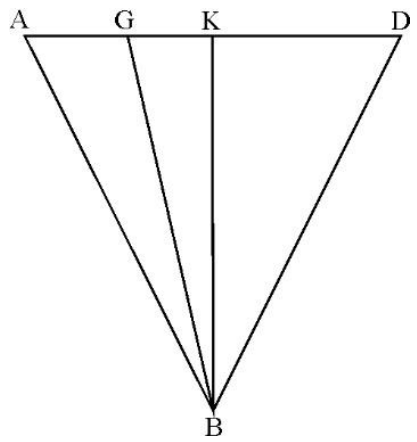

Figure 5. The figure in proposition 1 of the Optics.

For example, in the proposition 1 of the Optics, even if we took the figure to be directly a practical figure (see figure 5), and, in this case, AD a practical segment (and not "simply" a stroke that represents a geometrical segment by first representing a practical segment), the geometrical segment named (for us) AD does not represent a practical segment. It represents the spatial extension of the "thing seen" (BURTON, 1945, p. 357). This can be done since we can relate a practically straight extension (considering only its length) to a practical segment. So, while the geometrical segment is in a relation of idealization with a practical segment, the possibility of relating the practical segment to the spatial extension (e.g. in terms of measurements and possibly some approximations) enables to extend the representation relation existing between the geometrical segment and the practical segment to the spatial extension of a concrete object (the "thing seen").

\section{Conclusion}

RBHM, Vol. 20, n 39, pp. 13-33, 2020 
As we have seen, in ancient Greek practical geometry a concept like square corresponds directly to a drawn figure. This figure has practically equal sides making practically right angles between them. The equality of the sides can be seen as resulting from their measurement with, e.g., a rod or a rope corresponding to a particular unit of measure. In this way, the length of a segment is only meaningful as a measure; e.g., 2 feet. A practical figure like a square is drawn in a practically flat surface (a practical planar surface), even if this is left implicit. Otherwise, we could not maintain, e.g., that we draw a square. With pure geometry, we face a departure from the immediacy of practical geometry. There is no practically flat surface. What we have is its "counterpart", the Euclidean plane - which has a relation of idealization with the practically flat surface. This plane has several features not found in the practically flat surface. There is no clear indication of a boundary; it is something undefined or, at least, not mentioned. The Euclidean plane is a sort of "continuous" of absolute positions, where geometrical objects can be instantiated. A point, for which there is no measure (in the sense of pure geometry), i.e., which cannot be seen as a magnitude, when instantiated has an absolute position in the Euclidean plane (it is even difficult to talk about a point or any other geometrical object without considering them as instantiated in the Euclidean plane, since their properties are only fully established when considered as positioned in the plane). To address the relation of idealization of geometrical objects and practical figures, we considered, besides points, two other fundamental geometrical objects of ancient Greek planar geometry: segments (straight lines) and circles. These are conceived in terms of the idealization of their practical "counterparts". The idealizations are: the (geometrical) continuity, the "perfect" straightness of segments, the lack of depth, the breadthlessness, and the exactification of length. Afterward, we address how pure geometry "refers back" to practical geometry. As mentioned, we can have the case, e.g., that a geometrical segment having a relation of idealization with a practical segment, when applying pure geometry, is taken to represent a practical segment or a concrete extension. It is here that the to and fro between practical and pure geometry manifests at its most. There is another subtler way in which pure geometry "refers back" to practical geometry. This is, as we have seen, due to the figures accompanying in the propositions of pure geometry, in which, e.g., a practical segment represents a geometrical segment.

\section{References}

ABELL, C. 2009. Canny resemblance. In: The Philosophical Review, vol. 118, pp.183-223. ASPER, M. 2003. The two cultures of mathematics in ancient Greece. In: Robson, E., \& Stedall, J. (Eds.), The Oxford handbook of the history of mathematics. Oxford: Oxford University Press, pp. 107-132.

BÄCK, A. 2014. Aristotle's theory of abstraction. Heidelberg: Springer.

BARCELÓ, A. A. 2018. Mathematical pictures (draft version). Retrieved from <http://www.academia.edu/194 6205/Mathematical_Pictures> in May 2019. 
BLUMSON, B. 2014. Resemblance and representation. An essay in the philosophy of pictures. Cambridge: Open Book Publishers.

BURTON, H. E. 1945. The Optics of Euclid. In: Journal of the Optical Society of America, vol 35, pp. 357-372.

CUOMO, S. 2001. Ancient mathematics. London: Routledge.

EARMAN, J. 1989. World enough and space-time: absolute versus relational theories of space and time. Cambridge: Mit Press.

EUCLID 1956. The thirteen Books of the Elements (second edition, Vols. I-III). Translated with introduction and commentary by Sir Thomas L. Heath, from the critical edition of Heiberg. New York: Dover Publications.

HARARI, O. 2003. The concept of existence and the role of constructions in Euclid's Elements. In: Archive for History of Exact Sciences, vol 57, pp. 1-23.

HEATH, T. 1949. Mathematics in Aristotle. Oxford: Clarendon Press.

HØYRUP, J. 2002. Lengths, widths, surfaces: a portrait of Old Babylonian algebra and its kin. New York: Springer.

JAMME, M. 1993. Concepts of space. The history of theories of space in physics. Third, enlarged edition. New York: Dover Publications.

KNORR, W.R. 1986. The ancient tradition of geometric problems. Boston: Birkhäuser.

KULVICKI, J.V. 2014. Images. London: Routledge.

LEWIS, M.J.T. 2001. Surveying instruments of Greece and Rome. Cambridge: Cambridge University Press.

MUELLER, I. 1981. Philosophy of mathematics and deductive structure in Euclid's Elements. Cambridge: MIT Press.

NETZ, R. 1999. The shaping of deduction in Greek mathematics: a study in cognitive history. Cambridge: Cambridge University Press.

NETZ, R. 2004. Eudemus of Rhodes, Hippocrates of Chios and the earliest form of a Greek mathematical text. In: Centaurus, vol 46, pp. 243-286.

PLATO. 1997. Complete works. Edited with introduction and notes by J. M. Cooper. Indianapolis: Hackett Publishing Company.

TAISBAK, C. M. 2003. DEDOMENA. Euclid's Data or the importance of being given. Copenhagen: Museum Tusculanum Press.

\section{Mário Bacelar Valente}

Departamento de sistemas físicos, químicos e naturais - UPO - Sevilha - Espanha

E-mail: mar.bacelar@gmail.com 